\title{
IRREGULAR MANIFOLDS WHOSE CANONICAL SYSTEM IS COMPOSED OF A PENCIL *
}

\author{
JIN-XING $\mathrm{CAI}^{\dagger}$ AND ECKART VIEHWEG ${ }^{\ddagger}$
}

\begin{abstract}
Let $X$ be a complex projective $n$-dimensional manifold of general type whose canonical system is composite with a pencil. If the Albanese map is generically finite, but not surjective, or if the irregularity is strictly larger than $n$ and the image of $X$ in $\operatorname{Alb}(X)$ is of Kodaira dimension one, then the geometric genus $p_{g}(F)$ of a general fibre $F$ of the canonical map is one and the latter factors through the Albanese map. The last part of this result holds true for any threefold with $q(X) \geq 5$.
\end{abstract}

Introduction. In this note we study irregular higher dimensional complex projective manifolds $X$ whose canonical map $\Phi_{X}: X \rightarrow \mathbb{P}^{p_{g}(X)-1}$ has a one dimensional image. Hence replacing $X$ by some blowing up, the canonical map factors through a surjection $f: X \rightarrow C$ to a curve $C$ with connected fibres. We will call $f: X \rightarrow C$ the canonical fibration of $X$.

As shown by G. Xiao [X1] if the canonical system of a surface $S$ of general type is composite with a pencil, then $q(S) \leq 2$. Moreover this pencil is rational if $q(S)=2$.

Before formulating some generalizations to higher dimensional manifolds, let us recall that the Albanese dimension a $(X)$ of a manifold $X$ is defined to be the dimension of the image of the Albanese map alb $=\operatorname{alb}_{X}: X \rightarrow \operatorname{Alb}(X) . X$ is said to be of maximal Albanese dimension if $a(X)=\operatorname{dim}(X)$. Remark that the Kodaira dimension $\kappa(\operatorname{alb}(X))$ is always supposed to be the Kodaira dimension of a nonsingular model of $\operatorname{alb}(X)$.

Theorem 0.1 (Section 1 and Corollary 4.2). Let $X$ be a projective manifold of maximal Albanese dimension. Assume the canonical system is composite with a pencil, and let $f: X \rightarrow C$ be the canonical fibration, with general fibre $F$.

(i) If $q(X)>\operatorname{dim}(X)$, then

$$
\begin{aligned}
& p_{g}(X)=g(C) \geq 2, \quad q(X)=g(C)+\operatorname{dim}(X)-1, \quad \text { and } \quad \chi\left(\mathcal{O}_{X}\right)=0 . \\
& p_{g}(F)=1, \quad \text { and } \quad \chi\left(\mathcal{O}_{F}\right)=0 .
\end{aligned}
$$

(ii) If $q(X)=\operatorname{dim}(X)$, then $C \simeq \mathbb{P}^{1}$.

THEOREM 0.2 (Section 2). Let $X$ be a projective manifold whose canonical linear system is composite with a pencil. Assume that

$$
q(X)>\min \{\mathrm{a}(X)+1, \operatorname{dim}(X)\},
$$

and that $\kappa(\operatorname{alb}(X))=1$. Let $F$ denote the general fibre of the canonical fibration $f: X \rightarrow C$.

Then $p_{g}(F)=1$, and $f$ is the composition of $\operatorname{alb}_{X}$ with the Ueno map $u_{\operatorname{alb}(X)}$ (cf. (1.1)). Moreover $p_{g}(X)+1 \geq g(C) \geq 2$, and $q(X)=g(C)+\mathrm{a}(X)-1$.

*Received March 29, 2003; accepted for publication October 22, 2003. This work has been supported by the "DFG-Schwerpunktprogramm Globale Methoden in der Komplexen Geometrie" and by the DFG-NSFC Chinese-German project "Komplexe Geometrie". The first named author is also partially supported by the NSFC (No. 10271005) and SRF for ROCS, SEM.

${ }^{\dagger}$ LMAM, School of Mathematical Sciences and Institute of Mathematics, Peking University, Beijing 100871, P. R. China (cai@math.pku.edu.cn).

$\ddagger$ Universität Duisburg-Essen, FB6 Mathematik, 45117 Essen, Germany (viehweg@uni-essen.de). 
So the assumptions made in Theorem 0.1 , (i), and in Theorem 0.2 imply that the image $C$ of the canonical fibration $f$ is a curve positive genus, hence the universal property of the Albanese map implies that the canonical fibration factors through the Albanese map. For threefolds with $q(X) \geq 5$, one has a similar property.

TheOREM 0.3 (Section 3 ). Let $X$ be a threefold of general type, whose canonical linear system is composite with a pencil. Assume either one of the following conditions:

a. $q(X) \geq 5$.

b. $q(X) \geq 4$ and $X$ is of maximal Albanese dimension.

c. $q(X) \geq 4$ and $\operatorname{alb}(X)$ is a surface of Kodaira dimension 1.

d. $q(X) \geq 3$ and $\operatorname{alb}(X)$ is a curve.

Then the Stein factorization of the Albanese map $X \rightarrow \operatorname{alb}(X)$ factors through the canonical fibration.

Parts b), c) and d) in 0.3 are special cases of 0.1, i), and 0.2 , respectively, and obviously $0.1, \mathrm{i}$ ), implies that case b) never occurs (see 1.4). We do not know, whether the image of the canonical fibration can be $\mathbb{P}^{1}$ under the assumption a).

The bound $q(X) \geq 5$ is the best possible. In Section 3 we give examples of projective $n$-folds $X$ of general type with $q(X)=2 n-2$ and

$$
\kappa(\operatorname{alb}(X))=\mathrm{a}(X)=n-1,
$$

whose canonical fibration is a morphism $f: X \rightarrow \mathbb{P}^{1}$. If $h: X \rightarrow Y$ denotes the Stein factorization of $\operatorname{alb}_{X}$, then the induced map $X \rightarrow \mathbb{P}^{1} \times Y$ is generically finite. In those examples the geometric genus of a general fiber $F$ of $f$ is $p_{g}(F)=2^{n-1}+1>1$.

It is an open question whether there exists a constant $c(n)$, depending only $n$, such that for all $n$-dimensional manifolds with $q(X) \geq c(n), p_{g}(X) \geq 2$ and $a(X)<$ $\operatorname{dim}(X)$, the morphism $X \rightarrow Z \times \operatorname{alb}(X)$ induced by the Stein factorization $X \rightarrow Z$ of the canonical map and the Albanese map, is never surjective.

In Section 4 we will consider projective manifolds of general type and maximal Albanese dimension with $q(X)=\operatorname{dim}(X)$ whose canonical system is composite with a pencil (Examples 4.3 and 4.4 ).

For a projective variety $X^{\prime}$ we write $p_{g}\left(X^{\prime}\right)$, and $q\left(X^{\prime}\right)$ for the geometric genus and the irregularity of any smooth model $X$ of $X^{\prime}$, respectively. At several places we change the smooth birational model $X$. In particular we will always assume that the canonical map $\Phi_{X}$ is a morphism. We will say that the canonical fibration is a (rational / irrational) pencil, whenever the canonical linear system is composite with a pencil (and the image of the canonical fibration a rational / non-rational curve).

1. Manifolds of maximal Albanese dimension. Recall a well-known result on the geometry of subvarieties of an Abelian variety.

Proposition 1.1 (Ueno [Ue], Theorem 10.9, and Kollár, see [Mo], Corollary 3.10). Let $A$ be an Abelian variety, and $Y \subset A$ an irreducible reduced subvariety. Let $A_{0}$ be the connected component of $\{a \in A ; a+Y=Y\}$ containing the origin. Then $A_{0}$ is an Abelian subvariety of $A$, and

$$
u_{Y}: Y \longrightarrow B=Y / A_{0} \subset A / A_{0}
$$

is an étale fiber bundle with fiber $A_{0}$. Moreover $B$ is of general type, $u_{Y}$ is the Stein factorization of the canonical map of $Y$ and it is birational to the Iitaka fibration of $Y$. 
We will call $u_{Y}: Y \rightarrow B=Y / A_{0}$ the Ueno map.

For the proof of Theorem 0.1 we need two simple observations.

Proposition 1.2. Let $\alpha: X \rightarrow Y$ be a generically finite surjective morphism of complex projective manifolds. Then, $\omega_{Y}$ is a direct factor of $\alpha_{*} \omega_{X}$.

Proof. The statement is compatible with blowing up $X$ and $Y$, hence one may assume the branch locus $\Delta$ of $\alpha$ to be a normal crossing divisor. Let

$$
X \stackrel{g}{\longrightarrow} Z \stackrel{h}{\longrightarrow} Y
$$

be the Stein factorization of $\alpha$. Then $Z$ has at most quotient singularities and hence rational singularities (cf. e.g., [EV], Lemma 3.24), and $g_{*} \omega_{X}=\omega_{Z}$. Duality for finite morphisms (see [Ha], Ex. II 6.10, and [Ha], Ex. III 7.2) implies

$$
\alpha_{*} \omega_{X}=h_{*} \omega_{Z}=\mathcal{H o m}{ }_{Y}\left(h_{*} \mathcal{O}_{Z}, \omega_{Y}\right),
$$

so $\alpha_{*} \omega_{X}$ contains $\mathcal{H o m}_{Y}\left(\mathcal{O}_{Y}, \omega_{Y}\right)=\omega_{Y}$ as a direct factor.

LEMMA 1.3. Let $X$ be a projective manifold with $p_{g}(X) \geq 2$. Assume that the canonical fibration $f: X \rightarrow C$ is a pencil with general fiber $F$.

If $g(C) \geq 2$, then either $p_{g}(F)=1$ or $p_{g}(F)=p_{g}(X)=g(C)=2$.

Proof. Since $p_{g}(X) \neq 0$, we have $p_{g}(F)>0$. Assume that $p_{g}(F) \neq 1$. Since $\Phi_{X}$ factors through $f$, the moving part of $\left|K_{X}\right|$ is given by $f^{*} \mathcal{L}$ for some invertible subsheaf $\mathcal{L}$ of $f_{*} \omega_{X}$, splitting locally and of strictly positive degree; thus

$$
p_{g}(X)=h^{0}(\mathcal{L})=h^{0}\left(f_{*} \omega_{X}\right) \quad \text { and } \quad h^{0}\left(\omega_{C} \otimes \mathcal{L}^{-1}\right)<h^{0}\left(\omega_{C}\right) .
$$

Consider the exact sequence of sheaves

$$
0 \longrightarrow \mathcal{L} \longrightarrow f_{*} \omega_{X} \longrightarrow \mathcal{Q} \longrightarrow 0
$$

By the choice of $\mathcal{L}$ the sheaf $\mathcal{Q}$ is locally free of rank $p_{g}(F)-1$. By $[\mathrm{Fu}]$ the sheaf $f_{*} \omega_{X / C}=f_{*} \omega_{X} \otimes \omega_{C}^{-1}$ is nef, hence $\operatorname{deg}\left(\mathcal{Q} \otimes \omega_{C}^{-1}\right) \geq 0$. The Riemann-Roch Theorem for locally free sheaves on $C$ implies that

$$
\begin{aligned}
g(C)-1 & \geq h^{0}\left(\omega_{C} \otimes \mathcal{L}^{-1}\right)=h^{1}(\mathcal{L}) \geq h^{0}(\mathcal{Q}) \\
& \geq\left(p_{g}(F)-1\right)(g(C)-1) .
\end{aligned}
$$

Then $p_{g}(F)=2$ and $h^{1}(\mathcal{L})=g(C)-1$, hence $h^{0}(\mathcal{L})=\operatorname{deg}(\mathcal{L})$. By Clifford's Theorem $\operatorname{deg}(\mathcal{L})=2$ and $\mathcal{L} \simeq \omega_{C}$. So $p_{g}(X)=g(C)=2$.

Proof. [Proof of Theorem 0.1(i)] Blowing up $X$, if necessary, we may assume that the Albanese map alb $: X \rightarrow \operatorname{alb}(X) \subset \operatorname{Alb}(X)$ factors like

$$
X \stackrel{\alpha}{\longrightarrow} Y \stackrel{\delta}{\longrightarrow} \operatorname{alb}(X),
$$

for some desingularization $Y$ of $\operatorname{alb}(X)$. Let $\iota: Y \rightarrow B$ and $f: X \rightarrow C$ be the Stein factorizations of the canonical maps $\Phi_{Y}$ and $\Phi_{X}$, respectively. Proposition 1.1 and the assumption $q(X)>\operatorname{dim}(X)=\operatorname{dim}(Y)$ imply that $\operatorname{dim}(B) \geq 1$. On the other hand, by 1.2 there exists some torsion-free sheaf $\mathcal{G}$ with $\alpha_{*} \omega_{X}=\omega_{Y} \oplus \mathcal{G}$. So $\Phi_{Y} \circ \alpha$ is the composite of the canonical map $\Phi_{X}$, and a projection. In particular one obtains a surjection $C \rightarrow B$ and $B$ must be one dimensional. 
By $1.1 \iota: Y \rightarrow B$ is birational to an étale fibre bundle of Abelian subvarieties of $\operatorname{Alb}(X)$ of dimension $\operatorname{dim}(Y)-1=\operatorname{dim}(X)-1$, and

$$
q(X)=g(B)+\operatorname{dim}(X)-1 .
$$

On the other hand, the general fibre $F$ of $f$ is of maximal Albanese dimension, and its image in $\operatorname{Alb}(X)$ is an Abelian subvariety. Hence $q(X) \geq g(C)+\operatorname{dim}(F)$ and $g(C) \leq g(B)$. Since $C$ is a covering of $B$, this is only possible for $B=C$ and $g(C)=g(B) \geq 2$. Thus $f=\iota \circ \alpha$.

By Lemma 1.3, if $p_{g}(F) \neq 1$ one has

$$
p_{g}(F)=p_{g}(X)=g(C)=2 \quad \text { and } \quad q(X)=\operatorname{dim}(X)+1 .
$$

So $\operatorname{rank} f_{*} \omega_{X}=2$, and $\iota_{*} \mathcal{G}$ is an invertible sheaf. Since $f_{*} \omega_{X / C}$ is nef, $\iota_{*} \mathcal{G} \otimes \omega_{C}^{-1}$ is nef, and the Riemann-Roch Theorem implies $h^{0}\left(i_{*} \mathcal{G}\right)>0$, or

$$
p_{g}(X)=h^{0}\left(i_{*} \omega_{Y}\right)+h^{0}\left(i_{*} \mathcal{G}\right) \geq 3,
$$

a contradiction.

Since $F$ is of maximal Albanese dimension $p_{g}(F)=1$ implies that $q(F)=\operatorname{dim}(F)$ and $\chi\left(\mathcal{O}_{F}\right)=0$ (see $[\mathrm{Ue}]$ or $[\mathrm{Mo}]$, Cor. 3.4). The restriction map

$$
r: \operatorname{Pic}^{0}(X) \longrightarrow \operatorname{Pic}^{0}(F)
$$

is surjective. By the generic vanishing theorem ([GL] or $[\mathrm{EL}])$, for a general $\mathcal{L} \in$ $\operatorname{Pic}^{0}(X)$ the sheaf $\left.\omega_{F} \otimes \mathcal{L}\right|_{F}$ has no higher cohomology, hence

$$
h^{0}\left(\left.\omega_{F} \otimes \mathcal{L}\right|_{F}\right)=\chi\left(\left.\omega_{F} \otimes \mathcal{L}\right|_{F}\right)=0 .
$$

Tensoring the exact sequences

$$
0 \longrightarrow \omega_{X}(-(n+1) F) \longrightarrow \omega_{X}(-n F) \longrightarrow \omega_{F} \longrightarrow 0
$$

with $\mathcal{L}$, one obtains by descending induction on $n$ that $h^{0}\left(\omega_{X} \otimes \mathcal{L}\right)=0$. Using again the generic vanishing theorem $([\mathrm{GL}]$ or $[\mathrm{EL}])$ one finds $\chi\left(\mathcal{O}_{X}\right)=0$.

When $\operatorname{dim}(X)=3$, hence $\operatorname{dim}(F)=2$, the condition $\chi\left(\mathcal{O}_{F}\right)=0$ forces the Kodaira dimension of $F$ to be strictly smaller than two. By the easy addition formula for the Kodaira dimension one obtains an improvement of [Ca], Theorem 1.

Corollary 1.4. Let $X$ be a projective 3 -fold of general type and maximal Albanese dimension. If $q(X) \geq 4$, then the canonical linear system of $X$ is not composite with a pencil. In particular, if $p_{g}(X)=2$ then $q(X)=3$.

Corollary 1.5. Let $X$ be a projective manifold of maximal Albanese dimension. If $p_{g}(X)=2$, then $q(X) \leq \operatorname{dim}(X)+1$.

Proof. Let $f: X \rightarrow C$ be the canonical fibration. If $q(X)>\operatorname{dim}(X)+1$, Theorem 0.1 implies that $p_{g}(X)=g(C)=q(X)-\operatorname{dim}(X)+1 \geq 3$.

REMARK 1.6. In dimension greater than three there are manifolds $X$ satisfying the assumptions made in Theorem 0.1 or Corollary 1.5. For example, by [CH2] there exist smooth manifolds $F$ of general type and of maximal Albanese dimension with $p_{g}(F)=1$. If $C$ is a curve of genus $\geq 2$ then one can choose $X=F \times C$.

The arguments used to prove Lemma 1.3 can also be applied to bicanonical maps. 
THEOREM 1.7. Let $X$ be a projective manifold of maximal Albanese dimension, with $\kappa(X) \geq 2$ and $q(X)>\operatorname{dim}(X)$. Then the bicanonical linear system is not composite with a pencil.

Proof. Suppose that $\Phi_{2 X}$ is composite with a pencil, and let $h: X \rightarrow B$ be the fibration obtained as the Stein factorization of $\Phi_{2 X}$. A general fiber $F$ of $h$ has maximal Albanese dimension, and by the weak addition theorem $\kappa(F) \geq 1$, in particular $F$ is not an Abelian variety. By [CH1], Theorem 3.2, one finds $p_{2}(F) \geq 2$.

As in the proof of Lemma 1.3, there is an invertible subsheaf $\mathcal{L} \hookrightarrow h_{*} \omega_{X}^{\otimes 2}$, splitting locally and with $h^{0}(\mathcal{L})=p_{2}(X)$. The locally free quotient sheaf $\mathcal{Q}=h_{*} \omega_{X}^{\otimes 2} / \mathcal{L}$ is locally free of rank $p_{2}(F)-1$ and

$$
g(B)-1 \geq h^{0}\left(\omega_{B} \otimes \mathcal{L}^{-1}\right)=h^{1}(\mathcal{L}) \geq h^{0}(\mathcal{Q}) .
$$

By [V] the sheaf $h_{*} \omega_{X / B}^{\otimes 2}$ is nef, hence

$$
\operatorname{deg}(\mathcal{Q})-2\left(p_{2}(F)-1\right)(2 g(B)-2)=\operatorname{deg}\left(\mathcal{Q} \otimes \omega_{B}^{-2}\right) \geq 0
$$

The Riemann-Roch Theorem implies

$$
h^{0}(\mathcal{Q}) \geq 3\left(p_{2}(F)-1\right)(g(B)-1),
$$

and hence $g(B) \leq 1$.

By 1.1 and 1.2 one has $p_{g}(X) \geq 2$. The canonical map $\Phi_{X}$ is defined by a linear subsystem of $\left|K_{2 X}\right|$, hence it factors through $\Phi_{2 X}$, and the canonical fibration $f: X \rightarrow C$ factors through $h$. Since the fibres of $f$ and $h$ are both connected of the same dimension, $C=B$ is a curve of genus $\leq 1$, contradicting Theorem 0.1.

2. The case $\kappa(\operatorname{alb}(X))=1$.

Lemma 2.1. Let $X$ and $B$ be projective manifolds. Assume that the canonical fibration is a pencil $f: X \rightarrow C$, and let $h: X \rightarrow B$ be a morphism, such that $\alpha=(f, h): X \rightarrow C \times B$ is surjective. Then there is an injection

$$
\mathcal{O}_{B}^{\oplus p_{g}(X)} \longrightarrow h_{*} \omega_{X}
$$

Proof. Let us write $\alpha=(f, h): X \rightarrow C \times B$. Since $\Phi_{X}$ factors through $f$, we may choose again an invertible subsheaf $\mathcal{L}$ of $f_{*} \omega_{X}=p r_{1 *} \alpha_{*} \omega_{X}$ with $p_{g}(X)=h^{0}(\mathcal{L})$. Then $p r_{1}^{*} \mathcal{L}$ is a subsheaf of $\alpha_{*} \omega_{X}$ and one finds

$$
\mathcal{O}_{B}^{\oplus h^{0}(\mathcal{L})} \simeq p r_{2 *} p r_{1}^{*} \mathcal{L} \stackrel{\subset}{\longrightarrow} p r_{2 *} \alpha_{*} \omega_{X}=h_{*} \omega_{X}
$$

COROLlary 2.2. Let $X$ be a projective manifold, whose canonical linear system is composite with a pencil, and let $h: X \rightarrow B$ be a fiber space over a curve $B$ with $g(B) \geq 3$. Then $h$ factors through the canonical fibration $f: X \rightarrow C$.

Proof. If not, the induced map $\alpha=(f, h): X \rightarrow C \times B$ is surjective and 2.1 implies that $\operatorname{rank}\left(h_{*} \omega_{X}\right) \geq p_{g}(X)$. Using again the nefness of $h_{*} \omega_{X / B}$, the Riemann-Roch theorem implies that

$$
\begin{aligned}
p_{g}(X)=h^{0}\left(h_{*} \omega_{X}\right) & \geq \operatorname{deg}\left(h_{*} \omega_{X / B}\right)+\operatorname{rank}\left(h_{*} \omega_{X}\right)(g(B)-1) \\
& \geq p_{g}(X)(g(B)-1),
\end{aligned}
$$


for $g(B) \geq 3$ a contradiction.

Proof. [Proof of Theorem 0.2] By Theorem 0.1, we can assume that a $(X)<$ $\operatorname{dim}(X)$. Consider

$$
h=u \circ \text { alb }: X \stackrel{\text { alb }}{\longrightarrow} \operatorname{alb}(X) \stackrel{u}{\longrightarrow} B,
$$

where $u$ is the Ueno map. Then $B$ is a curve of genus

$$
g(B)=q(X)-\mathrm{a}(X)+1 \geq 3 .
$$

By Corollary $2.2 h$ factors through the canonical fibration $f: X \rightarrow C$. Then the image of a general fibre $F$ of $f$ under alb is an Abelian variety of dimension $a(X)-1$, hence $g(C) \leq q(X)-a(X)+1$, and $C=B$. By Lemma 1.3, one has $p_{g}(F)=1$. Since $f_{*} \omega_{X / C}$ is invertible and nef, the Riemann-Roch theorem implies that

$$
p_{g}(X)=h^{0}\left(f_{*} \omega_{X}\right) \geq \operatorname{deg}\left(f_{*} \omega_{X / C}\right)+g(C)-1 \geq g(C)-1 .
$$

REMARK 2.3. Examples, due to Beauville ([Be], Example 2), show that the condition on $q(X)$ in Theorem 0.2 can not be weakened.

There is a series of examples, showing that there are no further restrictions on the numerical invariants in 0.2 .

EXAMPLE 2.4. Let $T$ be a surface of general type with $p_{g}(T)=q(T)=0$ and $\pi_{1}(T)=\left\langle\sigma>\simeq \mathbb{Z}_{2}\right.$. Let $S \rightarrow T$ be the universal cover. Consider in addition a curve $\tilde{C}$ with an involution $\tau$ such that the genus of $C=\tilde{C} / \tau$ is $\geq 3$, and let $R$ be the ramification divisor of $\tilde{C}$ over $C$. Then

$$
X=(S \times \tilde{C}) / \sigma \times \tau
$$

is of general type with $p_{g}(X)=g(C)+\frac{1}{2} \operatorname{deg}(R)-1, q(X)=g(C)$, and

$$
\Phi_{X}=\operatorname{alb}_{X}: X \longrightarrow C=\tilde{C} / \tau
$$

is induced by the second projection.

As we will see in Section 3 , for $\operatorname{dim}(X)=3$ and $q(X)=4$ the condition on the Kodaira dimension of $\operatorname{alb}(X)$ in Theorem 0.2 is needed. Nevertheless one can show without this assumption:

Proposition 2.5. Let $X$ be a projective 3-fold of general type, whose canonical fibration $f: X \rightarrow C$ is a pencil, and let $F$ be a general fiber of $f$. If $q(X) \geq 4$ and $g(C) \geq 2$, then $p_{g}(F)=1$, and $f$ is the composite of $\operatorname{alb}_{X}$ and the Ueno map $u_{\mathrm{alb}(X)}$.

Proof. By Theorems 0.1 and 0.2, we may assume that a desingularization $S$ of $\operatorname{alb}(X)$ is a surface of general type. By Lemma 1.3, it only remains to exclude the case $g(C)=p_{g}(X)=p_{g}(F)=2$.

By the universal property of the Albanese map, there exists a morphism $h: S \rightarrow$ $C$. Since $f=h \circ$ albx, the fibres of $h$ are connected.

For a general point $p \in C$, consider the fibres $F=f^{*}(p)$, and $H=h^{*}(p)$. Since $X$ and $S$ are of general type, the easy addition formula implies that $F$ and $H$ are of general type. So

$$
2=p_{g}(F) \geq q(F) \geq g(H) \geq 2,
$$


and $q(F)=g(H)=2$. Then

$$
4 \leq q(X) \leq q(F)+g(C)=4 \quad \text { and } \quad q(S)=q(X)=4=g(H)+g(C) .
$$

This implies that $S \simeq C \times H$. Let $\alpha: X \rightarrow H$ be the composite of albx with the projection $S \rightarrow H$.

The sheaf $\mathcal{E}=\alpha_{*} \omega_{X / H}$ is nef. Assume that for $p \in H$ in general position

$$
H^{0}\left(\mathcal{E} \otimes \omega_{H} \otimes \mathcal{O}_{H}(-p)\right) \neq 0 .
$$

Then $\alpha^{-1}(p)$ belongs to the moving part of the linear system $\left|K_{X}\right|$, hence it must be a fibre of $f$, contradicting the construction of $\alpha$.

Using the Riemann-Roch theorem one finds

$$
0=h^{0}\left(\mathcal{E} \otimes \omega_{H} \otimes \mathcal{O}_{H}(-p)\right)=\operatorname{deg}(\mathcal{E})+h^{1}\left(\mathcal{E} \otimes \omega_{H} \otimes \mathcal{O}_{H}(-p)\right),
$$

and both, $\operatorname{deg}(\mathcal{E})$ and $h^{1}\left(\mathcal{E} \otimes \omega_{H} \otimes \mathcal{O}_{H}(-p)\right)$ are zero. The long exact cohomology sequence for

$$
0 \longrightarrow \mathcal{E} \otimes \omega_{H} \otimes \mathcal{O}_{H}(-p) \longrightarrow \mathcal{E} \otimes \omega_{H} \longrightarrow \mathbb{C}_{p}^{\operatorname{rank}(\mathcal{E})} \longrightarrow 0
$$

implies that $\operatorname{rank}(\mathcal{E})=p_{g}(X)=2$, hence $p_{g}\left(\alpha^{-1}(p)\right)=2$, and $\Phi_{X \mid \alpha^{-1}(p)}$ is the canonical map of $\alpha^{-1}(p)$. Then the image of the canonical fibration of $\alpha^{-1}(p)$ is a curve of genus $\geq 2$, contradicting $[\mathrm{X} 1]$.

3. $\operatorname{alb}(X)$ is of general type . As mentioned in the Introduction, Theorem 0.2 does not hold true, without the assumption $\kappa(\operatorname{alb}(X))=1$.

EXAMPLE 3.1. For $n \geq 3$ and

$$
\frac{3 n-3}{2} \leq q \leq 2 n-2,
$$

we will construct projective $n$-folds $X$ with irregularity $q(X)=q$ and with $\kappa(\operatorname{alb}(X))=\mathrm{a}(X)=n-1$, whose canonical linear system is composite with a rational pencil with connected fibres. To this aim, consider an étale double cover $\tilde{Y} \rightarrow Y=\tilde{Y} / \sigma$, satisfying the following condition:

(*) $Y$ is a manifold of general type, of maximal Albanese dimension, and with $p_{g}(\tilde{Y})=p_{g}(Y)+1$.

Let $C$ be a smooth curve of genus $g \geq 2$ with hyperelliptic involution $\tau$. Choose

$$
X=(\tilde{Y} \times C) / \sigma \times \tau,
$$

and $f: X \rightarrow \mathbb{P}^{1}=C / \tau$ to be the fibration induced by the second projection. Then $X$ is of general type with

$$
p_{g}(X)=g, \quad q(X)=q(Y), \operatorname{alb}_{X}(X)=\operatorname{alb}_{Y}(Y),
$$

and with $\Phi_{X}=f: X \rightarrow \mathbb{P}^{1}$. The product of the canonical fibration and the Albanese map factors like

$$
X \longrightarrow(\tilde{Y} / \sigma) \times(C / \tau)=\mathbb{P}^{1} \times Y \longrightarrow \mathbb{P}^{1} \times \operatorname{alb}_{Y}(Y),
$$

hence it is surjective.

To show that there exist examples of $\tilde{Y} \rightarrow Y$ satisfying the condition $(*)$ and with $q(Y)=q$ we start with: 
(i) $\operatorname{dim}(Y)=1$ : Consider an étale cover $\tilde{Y} \rightarrow Y$ of a curve $Y$ of genus 2 , hence $p_{g}(Y)=2, p_{g}(\tilde{Y})=3$, and $q(Y)=2$.

(ii) $\operatorname{dim}(Y)=2$ : Let $B$ be a non-hyperelliptic curve of genus 3 , let $Y=B^{(2)} \subset$ $\operatorname{Jac}(B)$ be the Theta divisor, and let $\alpha: \bar{A} \rightarrow \operatorname{Jac}(B)$ be the double cover defined by a non-zero 2-torsion element of $\operatorname{Pic}^{0} \operatorname{Jac}(B)$. Then the inverse image $\tilde{Y} \subset A$ of $Y$ under $\alpha$ is an étale double cover of $Y$, with $p_{g}(Y)=3$, $p_{g}(\tilde{Y})=4$ and $q(Y)=3$.

(iii) Assume that $\tilde{Y}_{i} \rightarrow Y_{i}(i=1,2)$ satisfies $(*)$, and let $\sigma_{i}$ be the corresponding involution. Then

$$
\tilde{Y}:=\left(\tilde{Y}_{1} \times \tilde{Y}_{2}\right) /\left(\sigma_{1} \times \sigma_{2}\right) \longrightarrow Y_{1} \times Y_{2}
$$

is an étale double cover with $p_{g}(\tilde{Y})=p_{g}\left(Y_{1}\right) p_{g}\left(Y_{2}\right)+1$ and $q(\tilde{Y})=q\left(Y_{1}\right)+$ $q\left(Y_{2}\right)$, hence it also satisfies $(*)$.

Now given $n \geq 3$ taking for $Y n-1$ copies of (i) we obtain examples with $q(X)=2 n-2$. If $n$ is odd, we may choose $\frac{n-1}{2}$ copies of (ii) and we find $X$ with $q(X)=\frac{3 n-3}{2}$. For $n$ even, one reaches $q(X)=\frac{3 n-2}{2}$. Of course, one can also find examples for all intermediate values.

Proof. [Proof of Theorem 0.3] Remark that part b) is a special case of Theorem 0.1 and that c) and d) are special cases of Theorem 0.2. Moreover, if the canonical fibration $f: X \rightarrow C$ is an irrational pencil, the Albanese map factors through $f$.

Hence it only remains to consider the case

$$
C=\mathbb{P}^{1}, \quad q(X) \geq 5, \quad \text { and } \quad \operatorname{dim}(\operatorname{alb}(X))=\kappa(\operatorname{alb}(X))=2 .
$$

Let

$$
X \stackrel{h}{\longrightarrow} S \longrightarrow \operatorname{alb}(X)
$$

be the Stein factorization of $\operatorname{alb}_{X}$. Blowing up $X$, if necessary, we will assume that $S$ is non-singular. Remark that $q(S)=q(X)$.

We will show step by step, that the surjectivity of

$$
\alpha=(f, h): X \longrightarrow \mathbb{P}^{1} \times S
$$

implies:

(i) The evaluation map $H^{0}\left(h_{*} \omega_{X}\right) \otimes \mathcal{O}_{S} \rightarrow h_{*} \omega_{X}$ is injective; in particular, $\operatorname{rank}\left(h_{*} \omega_{X}\right) \geq p_{g}(X)$.

(ii) $h^{0}\left(\Omega_{X}^{2}\right)=p_{g}(S)$.

(iii) $h^{i}\left(h_{*} \omega_{X}\right)=0$ for $i>0$.

(iv) $\chi\left(\mathcal{O}_{S}\right)=0$.

Of course, (iv) contradicts the assumption that $\operatorname{alb}(X)$, hence $S$, is a surface of general type.

(i) follows from Lemma 2.1 .

For (ii), replacing $S$ and $X$ by suitable smooth birational models, we will assume that for some simple normal crossing divisor $B$ on $S$ the morphism $h$ is smooth over $S \backslash B$, and that $\Delta=\left(h^{*} B\right)_{\text {red }}$ is a divisor with simple normal crossings. We have morphisms of sheaves

$$
h^{*} \Omega_{S}^{1} \stackrel{\subset}{\longrightarrow} \mathcal{E}=\Omega_{X}^{1} \cap h^{*} \Omega_{S}^{1}(\log B) \stackrel{\subset}{\longrightarrow} \Omega_{X}^{1} \stackrel{\epsilon}{\longrightarrow} \omega_{X / S}\left(\Delta-h^{*} B\right)
$$


where $\epsilon$ is the composite $\Omega_{X}^{1} \rightarrow \Omega_{X}^{1}(\log \Delta) \rightarrow \omega_{X / S}\left(\Delta-h^{*} B\right)$. Taking wedge product, one finds a complex of sheaves

$$
0 \longrightarrow h^{*} \omega_{S} \longrightarrow \Omega_{X}^{2} \longrightarrow \omega_{X / S} \otimes h^{*} \Omega_{S}^{1}
$$

Note that $h_{*}\left(\mathcal{O}_{X}\left(h^{*} B-\Delta\right)\right)=\mathcal{O}_{S}$, and that $h^{0}\left(h^{*} \omega_{S}\right)=h^{0}(\operatorname{det}(\mathcal{E}))$.

Now suppose that $h^{0}\left(\Omega_{X}^{2}\right)>p_{g}(S)$, choose $\varphi \in H^{0}\left(\Omega_{X}^{2}\right) \backslash h^{*} H^{0}\left(\omega_{S}\right)$, and let

$$
\mathcal{O}_{X} \longrightarrow \omega_{X / S} \otimes h^{*} \Omega_{S}^{1}
$$

be the induced non-trivial map. Tensoring (1) with $h^{*} \Omega_{S}^{1}$, one obtains

$$
h^{*} \Omega_{S}^{1} \longrightarrow \omega_{X / S} \otimes h^{*} \Omega_{S}^{1} \otimes h^{*} \Omega_{S}^{1} \longrightarrow \omega_{X / S} \otimes h^{*} \omega_{S}
$$

and, taking the direct image, one has a non-trivial map

$$
r: \Omega_{S}^{1} \longrightarrow h_{*} \omega_{X}
$$

Let $\mathcal{K}=\operatorname{Ker}\left(r: H^{0}\left(\Omega_{S}^{1}\right) \rightarrow H^{0}\left(h_{*} \omega_{X}\right)\right)$. Clearly

$$
\mathcal{K}=\left\{s \in H^{0}\left(\Omega_{S}^{1}\right) \mid s \wedge \varphi=0\right\} .
$$

Since $\operatorname{rank}(\operatorname{Im} r) \leq 2$, (i) implies that $h^{0}(\operatorname{Im} r) \leq 2$ and $\operatorname{dim}(\mathcal{K}) \geq q(S)-2$.

For a general point $x \in X$ define

$$
\Sigma=\left\{s \in \Omega_{x, X}^{1} \mid s \wedge \varphi=0\right\} .
$$

Clearly $\operatorname{dim}(\Sigma) \leq 2$, and by the choice of $\varphi$,

$$
\Sigma \not \subset h^{*} \Omega_{h(x), S}^{1}
$$

This implies that $h^{*} \mathcal{K}$ generates an invertible subsheaf of $h^{*} \Omega_{S}^{1}$, hence a morphism $S \rightarrow D$ to a curve $D$ of genus $g(D)=\operatorname{dim}(\mathcal{K}) \geq q(S)-2 \geq 3$, contradicting 2.2.

The Leray spectral sequence and the Serre duality for $h$ imply that

$$
h^{i}\left(\omega_{X}\right)=h^{i}\left(h_{*} \omega_{X}\right)+h^{i-1}\left(R^{1} h_{*} \omega_{X}\right)=h^{i}\left(h_{*} \omega_{X}\right)+h^{i-1}\left(\omega_{S}\right)
$$

Hence (iii) follows from $q(X)=q(S)$ and from (ii).

For (iv) remark first that (iii) together with semicontinuity, imply that for some open neighborhood $U \subset \operatorname{Pic}^{0}(S)$ of $\mathcal{O}_{S}$, and all $\mathcal{P} \in U$ one has

$$
H^{i}\left(h_{*} \omega_{X} \otimes \mathcal{P}\right)=0 \quad \text { for } \quad i>0 .
$$

It follows that for $\mathcal{P} \in U$,

$$
h^{0}\left(h_{*} \omega_{X} \otimes \mathcal{P}\right)=\chi\left(h_{*} \omega_{X}\right)=p_{g}(X) .
$$

Let $\mathcal{Q}$ be the cokernel of the injection $\mathcal{O}_{S}^{\oplus p_{g}} \rightarrow h_{*} \omega_{X}$ constructed in (i). For $P \in U$ one finds an exact sequence

$$
0 \rightarrow H^{0}\left(\mathcal{O}_{S}^{\oplus p_{g}} \otimes \mathcal{P}\right) \rightarrow H^{0}\left(h_{*} \omega_{X} \otimes \mathcal{P}\right) \rightarrow H^{0}(\mathcal{Q} \otimes \mathcal{P}) \rightarrow H^{1}\left(\mathcal{O}_{S}^{\oplus p_{g}} \otimes \mathcal{P}\right) \rightarrow 0
$$

an isomorphism

$$
H^{1}(\mathcal{Q} \otimes \mathcal{P}) \longrightarrow H^{2}\left(\mathcal{O}_{S}^{\oplus p_{g}} \otimes \mathcal{P}\right)
$$


and $H^{2}(\mathcal{Q} \otimes \mathcal{P})=0$. For $\mathcal{P}=\mathcal{O}_{S}$, one finds

$$
\begin{gathered}
h^{0}(\mathcal{Q})=h^{1}\left(\mathcal{O}_{S}^{\oplus p_{g}}\right)=p_{g}(X) q(X), \\
h^{1}(\mathcal{Q})=h^{2}\left(\mathcal{O}_{S}^{\oplus p_{g}}\right)=p_{g}(X) p_{g}(S), \\
h^{2}(\mathcal{Q})=0,
\end{gathered}
$$

whereas for $\mathcal{P} \neq \mathcal{O}_{S}$,

$$
\begin{gathered}
h^{0}(\mathcal{Q} \otimes \mathcal{P})=h^{0}\left(h_{*} \omega_{X} \otimes \mathcal{P}\right)=h^{0}\left(h_{*} \omega_{X} \otimes \mathcal{P}\right)=p_{g}(X), \\
h^{1}(\mathcal{Q} \otimes \mathcal{P})=h^{2}(\mathcal{Q} \otimes \mathcal{P})=0 .
\end{gathered}
$$

Since $\chi(\mathcal{Q})=\chi(\mathcal{Q} \otimes \mathcal{P})$, one obtains for the Euler characteristic

$$
\chi(\mathcal{Q})=p_{g}(X) q-p_{g}(X) p_{g}(S)=p_{g}(X) .
$$

Therefore, $q(S)=p_{g}(S)+1$, and hence $\chi\left(\mathcal{O}_{S}\right)=0$.

REMARK 3.2. In a first version of this note, we were not able to show that (i)(iii) implies (iv), hence not able to prove Theorem 0.3 , a), as it is stated now. The argument presented above, has been told us by Jungkai Alfred Chen.

4. The case $a(X)=q(X)=\operatorname{dim}(X)$.

Proposition 4.1. Let $X$ be a projective manifold whose canonical system is composite with a pencil, and let $f: X \rightarrow C$ be the canonical fibration. If the Albanese map alb $: X \rightarrow \operatorname{Alb}(X)$ is surjective, then either $C \simeq \mathbb{P}^{1}$, or $g(C)=1$ and $q(X)<$ $\operatorname{dim}(X)$. diagram

Proof. Clearly $g(C) \leq 1$. If $C$ is an elliptic curve, consider the commutative

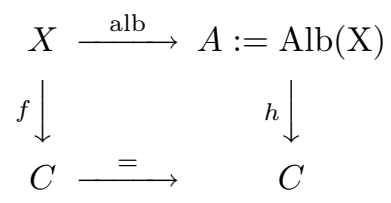

where $h$ is induced by the universal property of Albanese map. If $q(X)=\operatorname{dim}(X)$, Proposition 1.2 implies that $\mathrm{alb}_{*} \omega_{X}=\omega_{A} \oplus \mathcal{F}$ for some torsion-free sheaf $\mathcal{F}$ on $A$. Let $\mathcal{E} \subset f_{*} \omega_{X}=h_{*} \omega_{A} \oplus h_{*} \mathcal{F}$ be the subsheaf generated by the global sections of $f_{*} \omega_{X}$. Since $p_{g}(X)>p_{g}(A)=1$, one finds that $h^{0}(\mathcal{F})>0$, hence $\operatorname{rank}(\mathcal{E}) \geq 2$, contradicting the assumption that $\Phi_{X}$ factors through $f$. $\square$

Corollary 4.2. Let $X$ be a projective manifold, with $q(X)=\operatorname{dim}(X)$ and of maximal Albanese dimension. Assume the canonical system is composite with a pencil, and let $f: X \rightarrow C$ be the canonical fibration. Then $C \simeq \mathbb{P}^{1}$.

Let us end by giving some examples of manifolds $X$ of maximal Albanese dimension with $q(X)=\operatorname{dim}(X)$, and $p_{g}(X)$ arbitrarily large, whose canonical map is composite with a rational pencil.

ExAmple 4.3. Let $(A, \Theta)$ be a principally polarized Abelian $n$-fold $(n \geq 3)$, and $D \in|2 \Theta|$ a smooth divisor. The double cover obtained by taking the root out of $D$ is of general type with $p_{g}(X)=2$. 
EXAMPLE 4.4 (For $n=2$ see $[\mathrm{X} 2]$ ). For $n \geq 2$ let $(A, \Theta)$ be a simple polarized Abelian $n$-fold of type $(1, \cdots, 1,2)$. Let $\sigma: X^{\prime} \rightarrow A$ be a blowing up, such that the moving part of $\left|\sigma^{*} \Theta\right|$ defines a morphism $f^{\prime}: X^{\prime} \rightarrow C^{\prime}$, necessarily with $C^{\prime}=\mathbb{P}^{1}$. The general fibre $F^{\prime}$ of $f^{\prime}$ is of general type and $p_{g}\left(F^{\prime}\right)=h^{0}\left(\omega_{\Theta}\right)=n+1$. Since $p_{g}\left(X^{\prime}\right)=1$, one may write

$$
f_{*}^{\prime} \omega_{X^{\prime}}=\mathcal{O}_{\mathbb{P}^{1}} \oplus \mathcal{O}_{\mathbb{P}^{1}}\left(a_{1}\right) \oplus \cdots \oplus \mathcal{O}_{\mathbb{P}^{1}}\left(a_{n}\right),
$$

with $-1 \geq a_{1} \geq a_{2} \geq \ldots \geq a_{n}$. Since $f_{*}^{\prime} \omega_{X^{\prime} / \mathbb{P}^{1}}$ is nef by [Fu], $a_{n} \geq-2$. On the other hand, $\sigma_{*}\left(\omega_{X^{\prime}}\left(F^{\prime}\right)\right)$ is a subsheaf of $\mathcal{O}_{A}(\Theta)$, hence $h^{0}\left(\omega_{X^{\prime}}\left(F^{\prime}\right)\right) \leq 2$. One finds in $(2)$ $a_{1}=a_{2}=\cdots=a_{n}=-2$.

Let $\pi: C=\mathbb{P}^{1} \rightarrow C^{\prime}$ be a finite morphism of degree $d>1$, ramified only over points $p$ with $f^{\prime-1}(p)$ smooth, and let $f: X \rightarrow C$ be the pull-back family.

Base change and (2) imply that

$$
f_{*} \omega_{X / C}=\pi^{*} f_{*}^{\prime} \omega_{X^{\prime} / C^{\prime}}=\mathcal{O}_{\mathbb{P}^{1}}(2 d) \oplus \mathcal{O}_{\mathbb{P}^{1}}^{\oplus n},
$$

hence that $f_{*} \omega_{X}=\mathcal{O}_{\mathbb{P}^{1}}(2 d-2) \oplus \mathcal{O}_{\mathbb{P}^{1}}(-2)^{\oplus n}$. Then $p_{g}(X)=2 d-1$, the canonical map $\Phi_{X}$ factors through $f$, and $\omega_{X}$ contains $f^{*} \mathcal{O}_{\mathbb{P}^{1}}(2)$. The latter induces an inclusion $\omega_{X} / C \rightarrow \omega_{X}^{2}$, and since the general fiber of $f$ is of general type, $X$ must be of general type.

By construction $X$ is of maximal Albanese dimension, and Theorem 0.1 implies that $\operatorname{dim}(X)=q(X)=q\left(X^{\prime}\right)$. This of course follows by base change for $f_{*}^{\prime} \mathcal{O}_{X^{\prime}}$ and $R^{1} f_{*}^{\prime} \mathcal{O}_{X^{\prime}}$, as well.

Acknowledgments. Part of this work was done during the first author's stay at the Universität Essen. He thanks the members of the Department of Mathematics, in particular Hélène Esnault, for their help and hospitality. We are both very grateful to Jungkai Alfred Chen who realized that the vanishing of the higher cohomology of the sheaves $h_{*} \omega_{X}$ in the proof of Theorem 0.3 , a), can not hold true if $S$ is a surface of general type, and who allowed us to include his argument. This note has benefitted from several comments of the referee.

\section{REFERENCES}

[Be] A. Beauville, L'application canonique pour les surfaces de type général, Invent. Math., 55 (1979), pp. 121-140.

[Ca] J.-X. CAI, The Albanese map of a 3-fold of general type whose canonical map is composite with a pencil, Math. Zeitschrift, 240 (2002), pp. 511-519.

[CH1] J. A. Chen, C.D. HACON, Characterizations of Abelian varieties, Invent. Math., 143 (2001), pp. $435-447$.

[CH2] J. A. ChEn, C.D. HACON, On the irregularity of the image of the Iitaka fibration, preprint.

[EV] H. Esnault, E. Viehweg, Lectures on Vanishing Theorems, Birkh" auser Verlag Basel, 1992.

[EL] L. EIN, R. LAZARSFELD, Singularities of theta divisors and the birational geometry of irregular varieties, Jour. AMS, 10 (1997), pp. 243-258.

[Fu] T. Fujita, On Kaehler fibre spaces over curves, J. Math. Soc. Japan, 30 (1978), pp. 779-794.

[GL] M. Green, R. LaZArsfeld, Deformation theory, generic vanishing theorems, and some conjectures of Enriques, Catanese and Beauville, Invent. Math., 90 (1987), pp. 389-407.

[Ha] R. Hartshorne, Algebraic Geometry, GTM 52 Springer-Verlag, 1977.

[Ka] Y. Kawamata, Kodaira dimension of algebraic fiber spaces over curves, Invent. Math., 66 (1982), pp. 57-71.

[Mo] S. MorI, Classification of higher-dimensional varieties, Proceedings of Symposia in Pure Math., 46 (1987), pp. 269-331.

[Ue] K. Ueno, Classification theory of algebraic varieties and compact complex spaces, LNM 439, Springer-Verlag, 1975. 
[V] E. VIEHWEG, Weak positivity and the additivity of Kodaira dimension for certain fiber spaces, Adv. Stud. Pure Math., vol 1, North-Holland, 1983, pp. 329-353.

[X1] G. XIAO, L'irregularité des surfaces de type général dont le système canonique est composé d'un pinceau, Comp. Math., 56 (1985), pp. 251-257.

[X2] G. XIAO, Irregularity of surfaces with a linear pencil, Duke Math. J., 55 (1987), pp. 597-602. 